\title{
Recurrent Interstitial Pneumonitis and Pulmonary Hemorrhage Secondary to Amiodarone Toxicity
}

Rami Jambeih M.D. Victor Salloum M.D. Joe Lin M.D.

\section{Introduction}

Amiodarone is a commonly used drug with a wide range of toxicity. Pulmonary toxicity is among the most serious complications.

\section{Case Presentation}

* A 60-year-old male presented with dyspnea and hypoxemia.

* PMH includes atrial fibrillation for which he was started on Amiodarone 200 mg daily 9 months prior.

* CXR showed diffuse interstitial infiltrates.

* He failed a course of antibiotic treatment for 10 days.

* High resolution CT scan showed bilateral ground glass infiltrates suggestive of Amiodarone induced interstitial pneumonitis.

* Amiodarone was stopped and he was started on Prednisone.

* Symptoms improved at one-month follow-up and CXR infiltrates resolved.

- Prednisone was tapered and stopped after 2 weeks.

* One week later, he developed hypoxic respiratory failure with hemoptysis and bilateral lung infiltrates.

* Bronchoalveolar lavage revealed 3 bloody returns.

* Transbronchial biopsy showed fibrosis $(*)$, lipid laden (small arrows), and hemosiderin laden macrophages (large arrows) favoring the diagnosis of Amiodarone toxicity with diffuse alveolar hemorrhage.
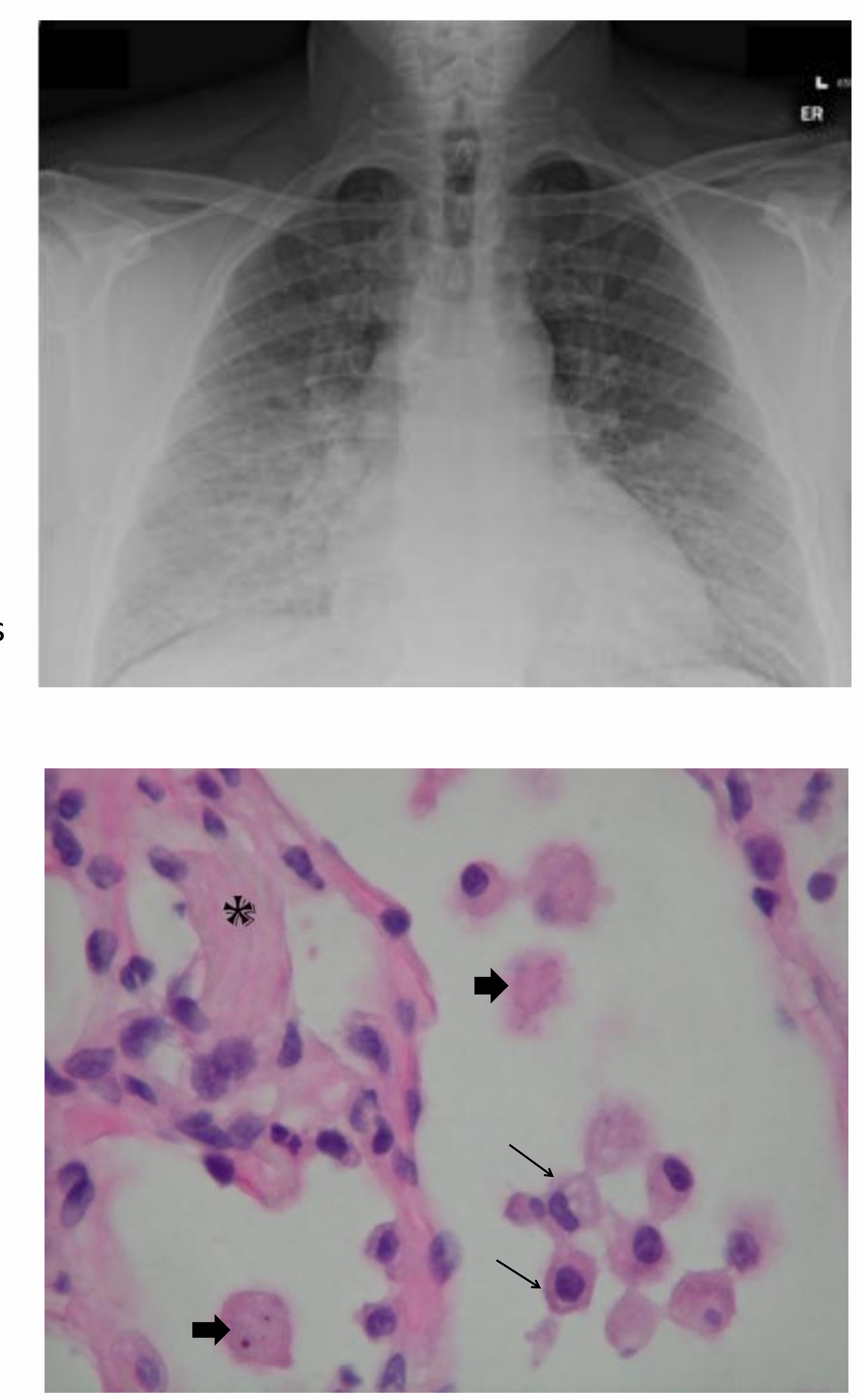

\section{Discussion}

* Pulmonary toxicity secondary to amiodarone use occurs in $5-15 \%$ of patients.

* Manifestations range from mild to severe and even fatal disease such as ARDS.

* Most common presentation is interstitial pneumonitis accounting for one-third of patients.

* Alveolar hemorrhage is a rare complication of amiodarone pulmonary toxicity. Only a few cases were reported.

* Amiodarone is a highly lipophilic drug that avidly binds to adipose tissues, resulting in a large distribution volume and a prolonged half-life reaching 180 days.

* Pulmonary toxicity may progress despite drug discontinuation.

Treatment includes stopping the offending drug and initiation of glucocorticoid therapy in severe cases.

* In our case, the rapid tapering of Prednisone apparently was responsible for the acute recurrence of a more severe form of interstitial pneumonitis with evidence of diffuse alveolar hemorrhage.

\section{Conclusion}

This case highlights the deleterious pulmonary side effects of Amiodarone and emphasizes the importance of slow tapering of glucocorticoids following amiodarone-induced lung injury.

\section{References}

1. Mason JW. Amiodarone. N Engl J Med 1987; 316:455-466.

2. Dean PJ, Groshart KD, Porterfield JG, lansmith DH, Golden EB Jr. Amiodarone-associated pulmonary toxicity. A clinical and pathologic study of eleven cases. Am J Clin Pathol 1987; 87:7-13.

3. Vizioli LD, Cho S. Amiodarone-associated hemoptysis. Chest 1994; 105:305- 306.

4. Podrid PJ. Amiodarone: Reevaluation of an old drug. Ann Intern Med 1995; 122:689-700.

5. Okayasu K, Takeda Y, Kojima J, Yoshizawa A, Kobayashi N, Sugiyama H, Kudo K. Amiodarone pulmonary toxicity: A patient with three recurrences of pulmonary toxicity and consideration of the probable risk for relapse. Intern Med 2006; 45:1303-1307.

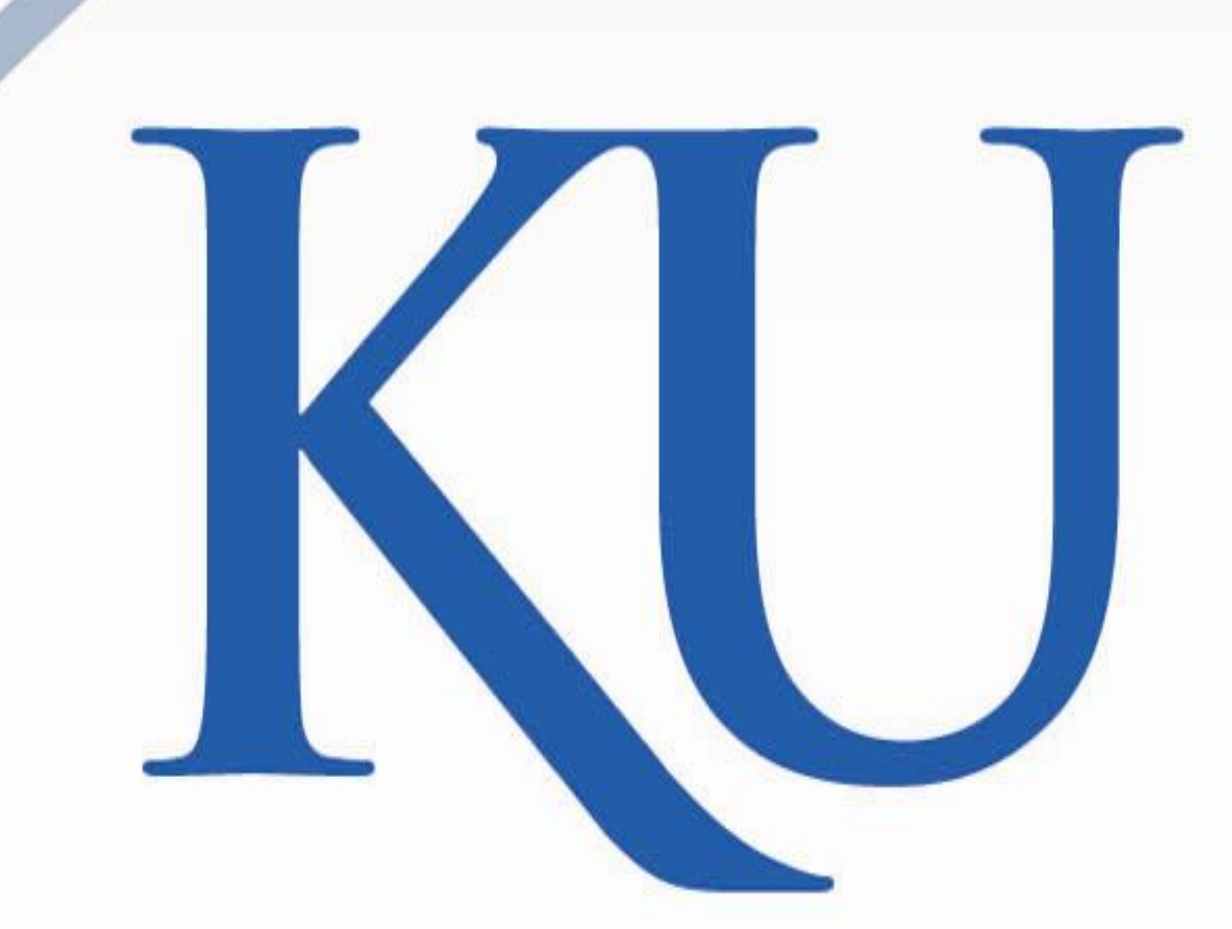

SCHOOL OF MEDICINE WICHTA

The University of Kansas 